\title{
Definition of the aortic valve plane by means of a novel dedicated software program: Proof of concept and validation with multi slice computed tomography
}

\author{
Nahid El Faquir ${ }^{1}$, Zouhair Rahhab ${ }^{1}$, Carl J Schultz ${ }^{1}$, Anne-Marie Maugenest ${ }^{1}$, Jean-Paul Aben ${ }^{2}$, \\ Tristan LB Slots ${ }^{2}$, Ron T van Domburg ${ }^{1}$, Nicolas Van Mieghem ${ }^{1}$, Peter PT de Jaegere ${ }^{1}$ \\ 1. Department of Cardiology, Erasmus MC, Rotterdam, The Netherlands. 2. Pie Medical Imaging, Maastricht, The \\ Netherlands
}

Correspondence: Peter PT de Jaegere. Address: Department of Cardiology, 's-Gravendijkwal 230, CE 3015, Rotterdam, The Netherlands. Email: p.dejaegere@erasmusmc.nl

Received: December 22, 2015 Accepted: January 25, $2016 \quad$ Online Published: January 26, 2016

DOI : $10.5430 /$ ijdi.v3n1p63

URL: http://dx.doi.org/10.5430/ijdi.v3n1p63

\section{Abstract}

Background: Proper selection of the $\mathrm{C}$-arm angulation during transcatheter aortic valve implantation (TAVI) is essential. Currently multi slice computed tomography (MSCT) is increasingly used to determine the C-arm angulation and is considered the gold standard. Yet, MSCT is not available in the catheterization laboratory and requires expertise. We sought to evaluate the accuracy of a novel software providing the optimal angiographic projection (i.e. three cusps on one line, equally in size and posterior, right and left cusp sequence- optimal angiographic projection [OAP]) based on two contrast angiograms.

Methods: Software was validated by comparing the difference in OAP between MSCT (reference) and software (CAAS A-Valve) in 67 patients and by qualitative comparison of the aortograms performed in accordance to MSCT and CAAS A-Valve defined OAP in 20 patients by 2 blinded assessors.

Results: The median (IQR) difference in the OAP between MSCT and software was 12.4 (7.5-20.1) and 18.9 (10.8-28.5) degrees. For the qualitative comparison, an overall score $\geq 2$ (acceptable-perfect) of $90 \%$ was reached by both observers for OAP derived by MSCT. It was $85 \%$ and $90 \%$ (observer 1 and 2 ) for OAP derived from the software and was $90 \%$ after consensus. The interobserver variability (mean difference and $S D$ ) for assessment of OAP curve and OAP itself was 1.6 $(S D=5.8, p=.23)$ and $9.5(S D=18.4)$ degrees $(p=.03)$ respectively.

Conclusions: The CAAS A-Valve software adequately defines the OAP and is clinically useful in patients who do not have a MSCT prior to TAVI.

\section{Keywords}

Transcatheter aortic valve implantation, Multi slice computed tomography, Aortic valve disease

\section{I ntroduction}

Transcatheter aortic valve implantation (TAVI) is increasingly used to treat selected patients with aortic stenosis and has shown to improve survival and quality of life ${ }^{[1-5]}$. Safety of the procedure depends on, among others, correct positioning of 
the bioprosthesis since inaccurate or incorrect positioning may lead to a number of complications such as conduction disorders, paravalvular leakage and valve embolization ${ }^{[6]}$. Proper selection of the $\mathrm{C}$-arm angulation in which the three aortic sinuses are depicted on one single line in the sequence of posterior, right and left sinus, the optimal angiographic projection (OAP), by contrast angiography during TAVI is essential ${ }^{[7-11]}$. Multi slice computed tomography (MSCT) is increasingly used to define OAP and is currently considered the gold standard ${ }^{[7,8,12]}$.

Yet, MSCT is not available in the catheterization laboratory and in absence of MSCT before TAVI repeated contrast angiograms have to be performed before valve implantation. To address this issue, software has been developed that allows the operator to select the optimal projection from a multitude of possible projections (OAP curve) but without certainty of the sequence consisting of posterior-, right-, and left coronary cusp ${ }^{[9]}$. The aim of the present study is to present a novel image analysis software (CAAS A-Valve 1.0, Pie Medical Imaging, the Netherlands) which provides the OAP based on two contrast angiograms with at least 30 degrees difference. We also sought to evaluate its accuracy by comparing the OAP derived from the software with the one derived from MSCT and its applicability by assessment of interobserver variability.

\section{Methods}

\subsection{Patients}

The study population consists of 67 patients with aortic stenosis who underwent TAVI between May 2007 and May 2012. All patients gave informed consent before admission for anonymised prospective data collection for clinical research purposes (data analysis and publication) and the study protocol conforms to ethical guidelines. All patients had tricuspid valves and undergone MSCT for procedure planning (i.e. access site and valve size) as described before ${ }^{[7]} .47$ patients were retrospectively identified who had two high quality aortography in two projections at least 30 degrees apart (cohort A). In these patients and in a prospective series of 20 patients (cohort B) in whom angiograms were performed in 2 predefined projections (LAO 50, RAO 25) the software was validated by comparison with MSCT. The OAP derived from the software was used for valve implantation if the one derived from MSCT was incorrect. In case the OAP derived from the software was also incorrect, conventional angiography with repeat injections was used to obtain an OAP.

\subsection{Determination of OAP by MSCT}

Technical details of the MSCT, protocol of data acquisition and analysis have been described in detail before ${ }^{[7,13,14]}$. The volume of iodinated contrast material (Visipaque ${ }^{\circledR} 320 \mathrm{mg} / \mathrm{ml}$, GE Health Care, Eindhoven, The Netherlands) was adapted to the expected scan time. A contrast bolus $(50-60 \mathrm{ml})$ was injected in an antecubital vein at a flow rate of $5.0 \mathrm{ml} / \mathrm{s}$ followed by a second contrast bolus of $30-40 \mathrm{ml}$ at $3.0 \mathrm{ml} / \mathrm{s}^{[13]}$.

\subsection{Determination of OAP by CAAS A-Valve}

OAP defined by the software was based upon the following: two aortograms (Iodixanol [Visipaque ${ }^{\mathrm{TM}}$ ] $20 \mathrm{ml}$, flow rate of $20 \mathrm{ml} / \mathrm{sec}$, cine speed of 30 frames/sec) which differ at least 30 degrees in viewing angle were first acquired. On both aortograms the ascending aorta is segmented by the user using spline drawing methods ${ }^{[15]}$ and a three-dimensional (3D) reconstruction of the aortic root is calculated based on well-established reconstruction techniques derived from CAAS QCA3D ${ }^{[16-18]}$. The 3D geometry of the aortic annulus is used to calculate all possible projections which are optimal in terms of viewing the aortic root perpendicular to the axial plane of the aortic valve annulus, resulting in an optimal angiographic projection curve (see Figure 1). Any projection on this curve is a projection in which all three aortic cusps are aligned (i.e. on one single line). On this curve there is one unique projection in which all three aortic cusps are viewed equally in size; the right coronary cusp R in-between the posterior cusp P (non-coronary cusp) and the left coronary cusp L, the OAP. To obtain this OAP, the user needs to identify the right coronary cusp in both aortograms. As soon as the right coronary cusp is indicated in one aortogram, guidance will be automatically provided to assist the user in selecting the 
right coronary cusp in the other aortogram. Based on this information the location of right coronary cusp on the surface of the $3 \mathrm{D}$ reconstruction is defined. Using the fact that the right coronary cusp is positioned in the centre of the aortic root, the OAP is calculated by computing the direction vector starting from the centre of the $3 \mathrm{D}$ reconstruction to the location of the right coronary cusp in the surface of the 3D reconstruction. Calculations were performed with CAAS A-Valve 1.0 (Pie Medical Imaging, the Netherlands).

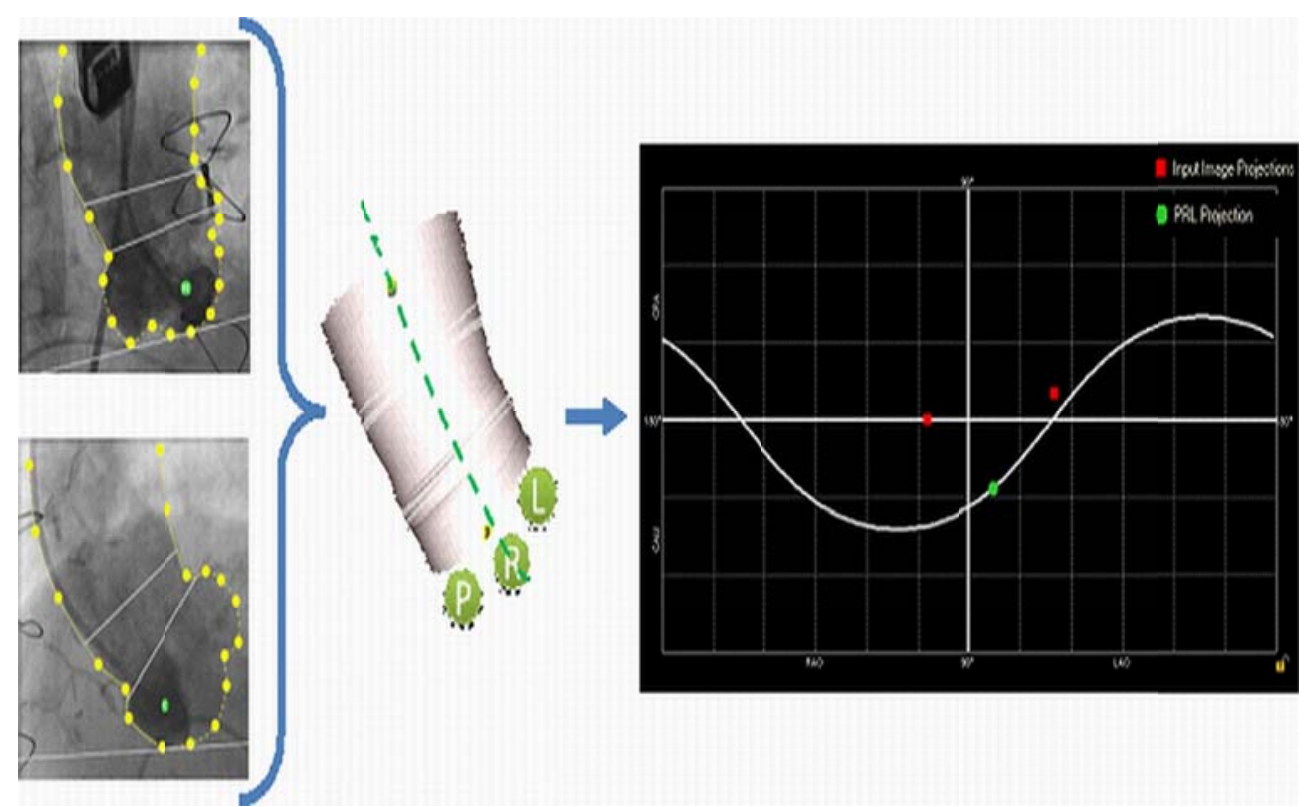

Figure 1. Schematic process in defining the optimal angiographic projection with CAAS A-Valve

\subsection{Comparison of OAP between MSCT and CAAS A-Valve}

The OAP's from MSCT and CAAS A-Valve were determined by independent and separate observers at separate times and blinded to one-another's results.

Validation of the accuracy of the software was performed by quantitative comparison of the OAP derived from MSCT and CAAS A-Valve in all patients (cohort A and B) by experts. This was done by calculating the 3D angle difference between MSCT and CAAS A-Valve projections as follows: The viewing direction of the x-ray source depends on the rotation and angulation of the $\mathrm{C}$-arm and defines a direction in space by a 3D unit

$$
\begin{gathered}
\text { vector }(x, y, z) ; \text { where } x=\sin (\text { rotation }) \cdot \cos (\text { angulation }), \\
y=\sin (\text { angulation }), \\
z=\cos (\text { rotation }) \cdot \cos (\text { angulation })
\end{gathered}
$$

To define the angle between two different X-ray viewing projections, the dot product is used of their unit vector. The angle between the MSCT projection and the OAP obtained by the software is calculated by:

$$
\text { angle }=\cos ^{-1}\left(x_{O A P} \cdot x_{M S C T}+y_{O A P} \cdot y_{M S C T}+z_{O A P} \cdot z_{M S C T}\right)
$$

Besides the OAP, the software also provides an optimal angiographic projection curve (OAP curve) representing all possible $\mathrm{C}$-arm projections in which the X-ray source is positioned perpendicular to the axial plane of the aortic valve annulus. The angle between the OAP derived from MSCT and the closest projection on this curve is defined by:

$$
\text { angle }=\text { ARGMIN }_{i} \cos ^{-1}\left(x_{i} \cdot x_{M S C T}+y_{i} \cdot y_{M S C T}+z_{i} \cdot z_{M S C T}\right)
$$


where $\left(x_{i}, y_{i}, z_{i}\right)$ is the 3D unit vector on each position $i$ of the optimal angiographic projection curve. Both angles are computed by use of Matlab 2007b (The MathWorks, Inc., Natick, Massachusetts, United States) (see Figure 2).

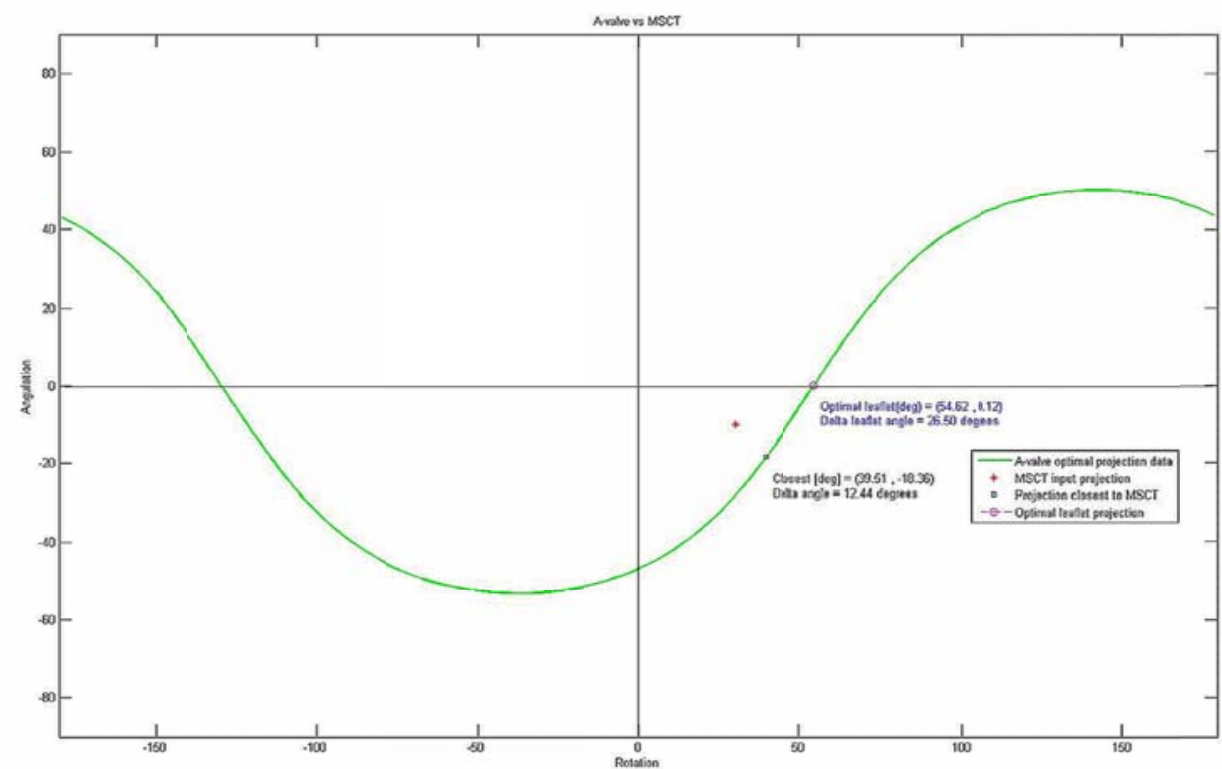

Figure 2. Example of Matlab output. An OAP curve is shown with the OAP on the curve. The MSCT projection and the closest point between the MSCT projection and the OAP curve are also shown

Qualitative comparison of the aortograms obtained in the OAP derived from MSCT (pre-procedure) and the CAAS A-Valve (peri-procedure) was made in cohort B by means of a scoring system in which the following 3 elements were visually assessed: 1) alignment of aortic sinuses on one single line, 2) unique sequence of sinuses and 3) an overall score revealing the degree of physician's satisfaction of the proposed OAP (see Figure 3 ). The OAP images were independently scored by two operators using a score varying between 1 and 3 (i.e. $3=$ perfect, $2=$ acceptable and $1=$ poor). In case of discrepancy, consensus was reached by plenary discussion with the 2 operators. Discrepancy was defined as a score difference of two points in one of the three elements.

Figure 3. Illustration of visual assessment and grading of optimal projection

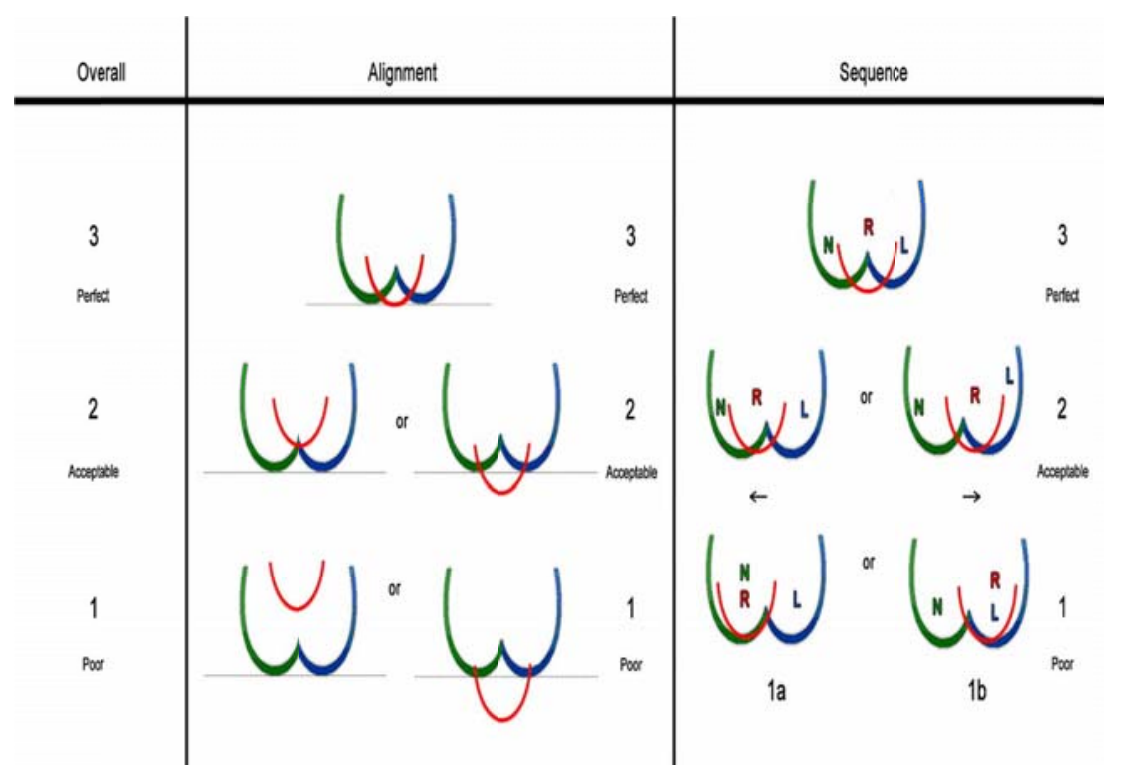

ISSN 2331-5857 E-ISSN 2331-5865 
Validation of the reproducibility of the software was performed in cohort B. Two independent investigators, which were trained in using the CAAS A-Valve software, performed an analysis of all patients within cohort B. Reproducibility of the OAP was assessed by the interobserver variability of the 3D angle between the OAP obtained by two observers using the mathematical dot product;

$$
\text { angle }=\cos ^{-1}\left(x_{o b s 1} \cdot x_{o b s 2}+y_{o b s 1} \cdot y_{o b s 2}+z_{o b s 1} \cdot z_{o b s 2}\right)
$$

In order to define the sign of the angle we choose as reference vector the mean normal vector of the OAP's from observer 1 and observer 2 obtained in each patient.

The reproducibility of the OAP curve was measured by computing the difference in the normal vector of the plane defining the optimal projection (see Figure 4) between the two observers using the mathematical dot product. The plane defining the optimal projection was reconstructed from the OAP curve. Sign differences ( + or -$)$ were defined by using a reference vector which was perpendicular to the mean normal vector of all measurements from observer 1 and observer 2 .

Figure 4. Illustration of the normal vector of the optimal valve plane

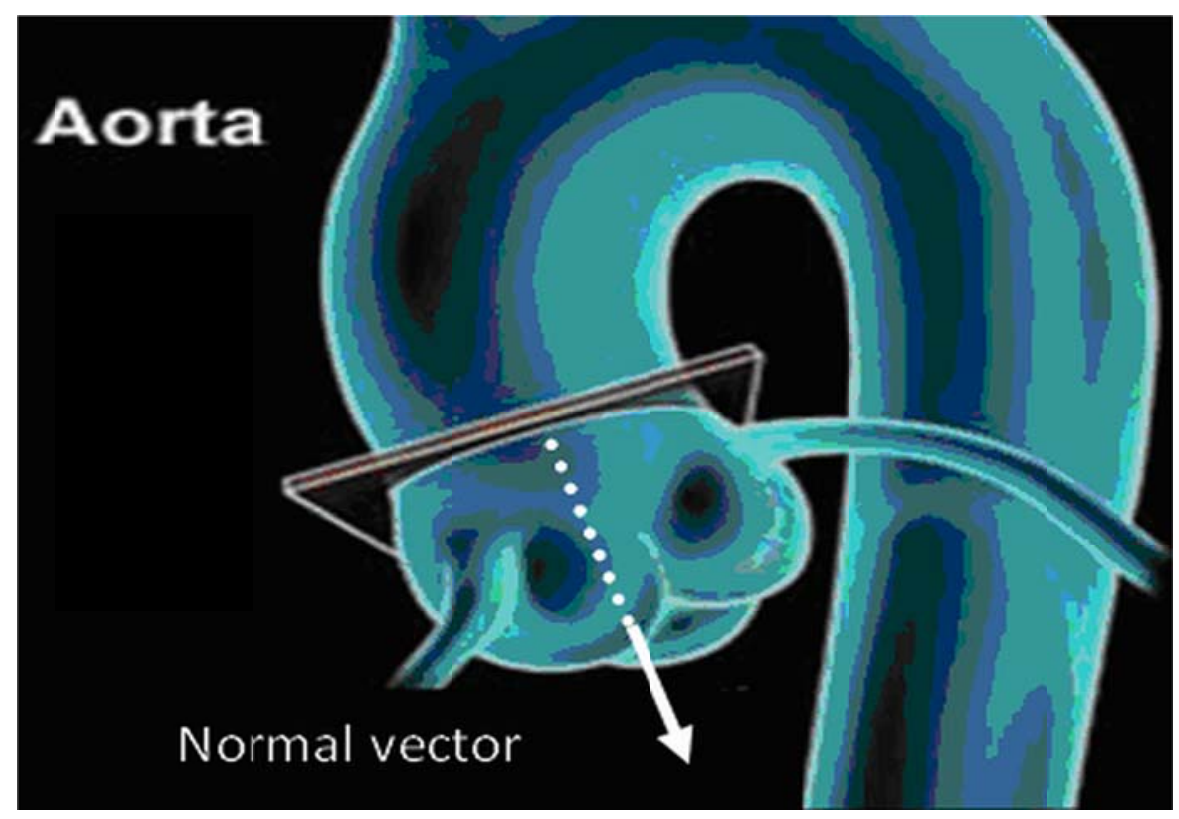

\subsection{Statistics}

Categorical variables are presented as frequencies and percentages, continuous variables are presented as the mean and $S D$ or, if the distribution was not Gaussian, as the median and interquartile range (IQ range). For the reproducibility a signed Student's $t$-test was performed. A $P$ value of less than .05 was considered to indicate a statistically significant difference. Statistical analysis was done using SPSS 20.0.

\section{Results}

The baseline patient characteristics and procedural information are summarized in Table 1. With respect to validation, the median (IQR) difference in the OAP between MSCT and the software was 12.4 (7.5-20.1) and 18.9 (10.8-28.5) degrees in cohort A and B, respectively. The median (IQR) difference between the OAP derived from MSCT and the nearest point on the OAP software curve was 8.8 (3.9-17.7) and 14.6 (9.4-17.9) degrees in cohort A and B, respectively (see Figure 2). 
Table 1. Baseline and procedural characteristics $(n=67)$

\begin{tabular}{|l|l|}
\hline Age $(\mathrm{yrs})$, median $(\mathrm{IQR})$ & $81(80-86)$ \\
\hline Male, $\mathrm{n}(\%)$ & $39(58)$ \\
\hline Height $(\mathrm{cm})$, mean $\pm S D$ & $167 \pm 9$ \\
\hline Weight $(\mathrm{kg})$, median $(\mathrm{IQR})$ & $72(65-86)$ \\
\hline Body Mass Index $\left(\mathrm{kg} / \mathrm{m}^{2}\right)$, median $(\mathrm{IQR})$ & $27(24-29)$ \\
\hline Body Surface Area $\left(\mathrm{m}^{2}\right)$, median $(\mathrm{IQR})$ & $1.83(1.69-2.05)$ \\
\hline Tricuspid valve, $\mathrm{n}(\%)$ & $67(100)$ \\
\hline Valve type, $\mathrm{n}(\%)$ & \\
\hline Medtronic Corevalve System & $64(96)$ \\
\hline Edwards Sapien & $2(3)$ \\
\hline No valve & $1(1)$ \\
\hline Valve size, $\mathrm{n}(\%)$ & \\
\hline 26 & $24(36)$ \\
\hline 29 & $38(57)$ \\
\hline 31 & $4(6)$ \\
\hline
\end{tabular}

With respect to the qualitative comparison of OAP between MSCT and software, an overall score $\geq 2$ (acceptable or perfect) of $90 \%$ was reached by both observers for OAP derived by MSCT. It was $85 \%$ and $90 \%$ (observer 1 and 2 ) for OAP derived from the software and was 90\% after consensus (see Figure 5). For alignment of the sinuses by MSCT, a score $\geq 2$ (acceptable or perfect) was $85 \%$ and $90 \%$ in observer 1 and 2 , respectively and, was $95 \%$ and $85 \%$ for the software. After consensus, a score of $90 \%$ was reached for both MSCT and the software. For the unique sequence of the sinuses by MSCT, a score $\geq 2$ (acceptable or perfect) was $85 \%$ in both observers and was $80 \%$ and $85 \%$ by the software in observer 1 and 2 . After consensus, a score of $95 \%$ was reached for MSCT and $85 \%$ for the software.

Observer 1 before consensus

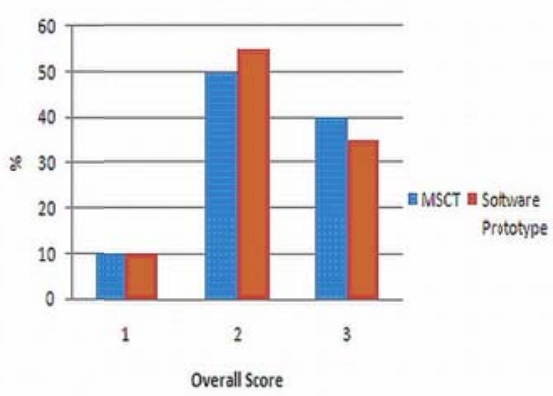

Observer 2 before consensus

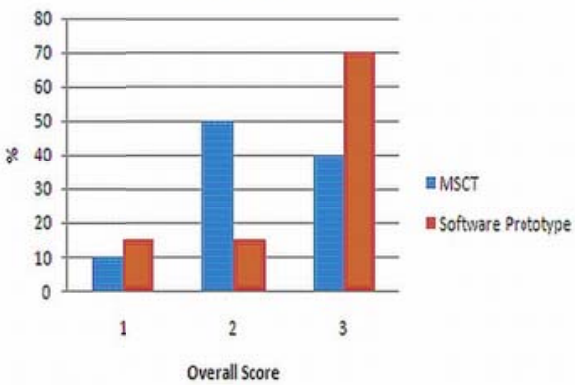

Observer 1 after consensus

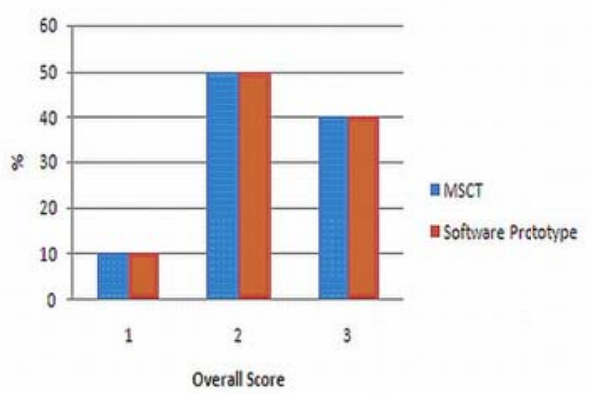

Observer 2 after consensus

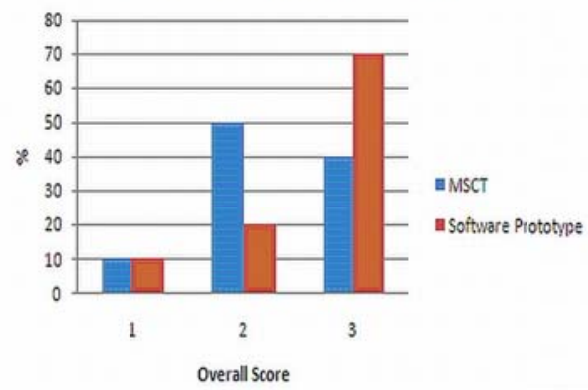

Figure 5. Details of the qualitative comparison of OAP between MSCT and software concerning the overall score 
The mean $(S D)$ interobserver variability (applicability software) for assessment of OAP curve and OAP itself was 1.6 $(S D=5.8, p=.23)$ and $9.5(S D=18.4)$ degrees $(p=.03)$ respectively.

During valve implantation in 8 cases the OAP derived from the software was used, in 8 cases the OAP derived from MSCT was used and in 4 cases the OAP derived by conventional angiography with repeat injections was used.

\section{Discussion}

In this study, software for the definition of the OAP is described. We found that the CAAS A-Valve software accurately determines OAP in comparison with MSCT. The median (IQR) difference in OAP between the software and MSCT was 12.4 (7.5-20.1) and 18.9 (10.8-28.5) degrees in cohort A and B, respectively. Qualitatively, the overall score and alignment were found to be equal for both the software and MSCT. MSCT, however, was marginally superior to the software in the definition of the unique sequence of sinuses.

The importance of appropriate visualization of the aortic root in the various phases of TAVI (planning, execution and evaluation) is now widely recognized and has been subject of clinical research evaluating novel software for improved visualization of the aortic root including valve plane and on-line continuous assessment of the relation between the bioprosthesis and annulus ${ }^{[8-11,19-22]}$.

With respect to clinical relevance of OAP, Samim et al. compared OAP derived from pre-TAVI MDCT by a dedicated automated 3D analysis system with conventional angiography and demonstrated that the first mentioned is associated with a reduction in contrast use, radiation and less kidney injury during TAVI ${ }^{[10]}$. In contrast to Samim et al., CAAS A-Valve requires two angiograms' and does not depend on a pre TAVI CT scan or conventional angiography with repeat injections, which could be also associated with less radiation exposure, less total contrast use and less kidney injury. Since CAAS A-Valve is not based on a pre TAVI CT scan, there is also no necessity of a MSCT scanner and its expertise. Furthermore, the automated 3D analysis system described by Samim et al. may affect its performance since the position of the patient during MSCT may vary from the one during TAVI. Also Gurvitch et al., used pre-TAVI MSCT which was evaluated in a cohort with only conventional angiography which resulted in no difference in outcome ${ }^{[8]}$. However, MSCT seemed to provide more accurate valve deployment and reduce malposition, this needs to be confirmed for CAAS A-Valve in future studies. An important difference is that angiographic separation and correct sequence of the sinuses was a requirement of the definition of the OAP in the present study, but not in earlier studies ${ }^{[8,10]}$. When this requirement is not specifically addressed, the non- and right coronary sinuses may completely overlap in some patient anatomies in commonly used implantation views, which may lead to inadvertent positioning of the pigtail catheter in the right instead of the non-coronary sinus. As the pigtail is sometimes used as reference point to guide the device positioning before deployment this could lead to device release at a higher level than intended by the operator.

In Kurra et al., a comparison between angiography and MDCT demonstrated no significant difference in annulus diameter in the LAO projection, respectively $2.3 \mathrm{~cm}(S D=0.3) v s .2 .4 \mathrm{~cm}(S D=0.3),(p=.052 ; 95 \% \mathrm{CI}:-0.1$ to 0.2$)$ and a small but significant difference in the RAO projection, respectively $2.4 \mathrm{~cm}(S D=0.3) v s .2 .2 \mathrm{~cm}(S D=0.3),(p=.029 ; 95 \% \mathrm{CI}:-0.2$ to 0.01$)^{[23]}$. In contrast with Kurra et al., a comparison between the complete angulations (in degrees) of the OAP derived by MSCT and CAAS A-Valve is made here instead of an apart analyses of the RAO and LAO angles based on annulus diameter. Due to the difference in analyses, a comparison with the present study is not likely and this may also explain the difference in the quantitative measurements. Moreover, determination of OAP in clinical practice consist of qualitative elements which cannot be assessed by numbers.

Similar to our study, the software described by Tzikas et al. does not depend on a pre-TAVI MSCT ${ }^{[9]}$. Yet, the software described by Tzikas et al. provides only the OAP curve while CAAS A-Valve provides both the OAP curve and the ideal working projection (OAP). The latter may be associated with lesser contrast use and safety by improved implantation 
technique as a result of optimal visualisation of the target zone. The clinical benefit of OAP relative to other techniques of planning and guidance, however, needs to be assessed by appropriately designed studies.

Both MSCT and CAAS A-valve derived OAP's were accurate enough to be clinically useful in $90 \%$ of cases. Yet the cases where accuracy was suboptimal were different for MSCT and the software, likely because of the differences between the 2 techniques. The MSCT definition of the OAP may be hindered in cases with substantial calcification at the level of the annular plane, due to the calcium blooming effect caused by signal attenuation. In contrast, the software derived OAP is not much hindered by the presence of calcium, but relies on 3D reconstruction which may sometimes vary from the true 3D geometry in patients with unusual anatomy or if the tracing of the aortic outline by the operator is inaccurate. The high clinical utility of both MSCT and software derived OAP's, despite angular differences between the 2 techniques, indicates that there is a range of projections close to the OAP curve that meet the clinical requirements of the OAP, rather than only a single projection. Both techniques provide an accurate practical solution in the vast majority of patients without the need for additional projections.

\section{Limitations}

The present study is a proof of concept of which its applicability and clinical benefit needs to be confirmed. MSCT usually requires more contrast than aortography for determining the OAP $(80-100 \mathrm{ml} v \mathrm{~s} .40 \mathrm{ml})$, while it provides additional information to guide vascular access and sizing. Therefore, the value of OAP may be restricted in situations where there is no availability of MSCT.

\section{Conclusion}

Whereas MSCT should be used to determine the OAP in patients where it is available for procedure planning, the CAAS A-Valve software is clinically useful in patients who do not have a MSCT prior to TAVI, or when the expertise needed to obtain the OAP from MSCT is not available.

\section{References}

[1] Mylotte D, Osnabrugge RL, Windecker S, et al. Transcatheter aortic valve replacement in Europe: adoption trends and factors influencing device utilization. J Am Coll Cardiol. 2013; 62(3): 210-9. http://dx.doi.org/10.1016/j.jacc.2013.03.074

[2] Leon MB, Smith CR, Mack M, et al. Transcatheter aortic-valve implantation for aortic stenosis in patients who cannot undergo surgery. N Engl J Med. 2010; 363(17): 1597-607. http://dx.doi.org/10.1056/NEJMoa1008232

[3] Smith CR, Leon MB, Mack MJ, et al. Transcatheter versus surgical aortic-valve replacement in high-risk patients. N Engl J Med. 2011; 364(23): 2187-98. http://dx.doi.org/10.1056/NEJMoa1103510

[4] Kodali SK, Williams MR, Smith CR, et al. Two-year outcomes after transcatheter or surgical aortic-valve replacement. N Engl J Med. 2012; 366(18): 1686-95. http://dx.doi.org/10.1056/NEJMoa1200384

[5] Makkar RR, Fontana GP, Jilaihawi H, et al. Transcatheter aortic-valve replacement for inoperable severe aortic stenosis. N Engl J Med. 2012; 366(18): 1696-704. http://dx.doi.org/10.1056/NEJMoa1202277

[6] Leipsic J, Yang TH, Min JK. Computed Tomographic Imaging of Transcatheter Aortic Valve Replacement for Prediction and Prevention of Procedural Complications. Circulation: Cardiovascular Imaging. 2013; 6(4): 597-605. http://dx.doi.org/10.1161/CIRCIMAGING.113.000334

[7] Schultz C, Moelker A, Tzikas A, et al. The use of MSCT for the evaluation of the aortic root before transcutaneous aortic valve implantation: the Rotterdam approach. EuroIntervention. 2010; 6(4): 505-11. http://dx.doi.org/10.4244/EIJ30V6I4A84

[8] Gurvitch R, Wood DA, Leipsic J, et al. Multislice computed tomography for prediction of optimal angiographic deployment projections during transcatheter aortic valve implantation. JACC Cardiovasc Interv. 2010; 3(11): 1157-65.

http://dx.doi.org/10.1016/j.jcin.2010.09.010 
[9] Tzikas A, Schultz C, Van Mieghem NM, et al. Optimal projection estimation for transcatheter aortic valve implantation based on contrast-aortography: validation of a Prototype Software. Catheter Cardiovasc Interv. 2010; 76(4): 602-7. http://dx.doi.org/10.1002/ccd.22641

[10] Samim M, Stella PR, Agostoni P, et al. Automated 3D analysis of pre-procedural MDCT to predict annulus plane angulation and C-arm positioning: benefit on procedural outcome in patients referred for TAVR. JACC Cardiovasc Imaging. 2013; 6(2): 238-48. http://dx.doi.org/10.1016/j.jcmg.2012.12.004

[11] Kasel AM, Cassese S, Leber AW, et al. Fluoroscopy-guided aortic root imaging for TAVR: "follow the right cusp" rule. JACC Cardiovasc Imaging. 2013; 6(2): 274-5. http://dx.doi.org/10.1016/j.jcmg.2012.06.014

[12] Nguyen G, Leipsic J. Cardiac computed tomography and computed tomography angiography in the evaluation of patients prior to transcatheter aortic valve implantation. Current Opinion in Cardiology. 2013; 28(5): 497-504. http://dx.doi.org/10.1097/HCO.0b013e32836245c1

[13] Schultz CJ, Moelker A, Piazza N, et al. Three dimensional evaluation of the aortic annulus using multislice computer tomography: are manufacturer's guidelines for sizing for percutaneous aortic valve replacement helpful? Eur Heart J. 2010; 31(7): 849-56. http://dx.doi.org/10.1093/eurheartj/ehp534

[14] Schultz CJ, Weustink A, Piazza N, et al. Geometry and degree of apposition of the CoreValve ReValving system with multislice computed tomography after implantation in patients with aortic stenosis. J Am Coll Cardiol. 2009; 54(10): 911-8. http://dx.doi.org/10.1016/j.jacc.2009.04.075

[15] Browne MA, Gaydecki PA. High-speed spline fitting, with application to boundary tracing in low-contrast digital images. Comput Biol Med. 1987; 17: 109-116. http://dx.doi.org/10.1016/0010-4825(87)90005-9

[16] Schuurbiers JC, Lopez NG, Ligthart J, et al. In vivo validation of CAAS QCA-3D coronary reconstruction using fusion of angiography and intravascularultrasound (ANGUS). Catheter Cardiovasc Interv. 2009; 73(5): 620-6. http://dx.doi.org/10.1002/ccd.21872

[17] Girasis C, Schuurbiers JC, Muramatsu T, et al. Advanced three-dimensional quantitative coronary angiographic assessment of bifurcation lesions: methodologyand phantom validation. EuroIntervention. 2013; 8(12): 1451-60. http://dx.doi.org/10.4244/EIJV8I12A219

[18] Onuma Y, Girasis C, Aben JP, et al. A novel dedicated 3-dimensional quantitative coronary analysis methodology for bifurcation lesions. Eurointervention. 2011; 7(5): 629-35. http://dx.doi.org/10.4244/EIJV7I5A100

[19] Marwick TH, Narula J. The expanding frontier in valve imaging. JACC Cardiovasc Imaging. 2013; 6(2): 281-2. http://dx.doi.org/10.1016/j.jcmg.2013.01.002

[20] Watanabe Y, Morice MC, Bouvier E, et al. Automated 3-dimensional aortic annular assessment by multidetector computed tomography in transcatheter aortic valve implantation. JACC Cardiovasc Interv. 2013; 6(9): 955-64. http://dx.doi.org/10.1016/j.jcin.2013.05.008

[21] Dvir D, Lavi I, Eltchaninoff H, et al. Multicenter evaluation of Edwards SAPIEN positioning during transcatheter aortic valve implantation withcorrelates for device movement during final deployment. JACC Cardiovasc Interv. 2012; 5(5): 563-70. http://dx.doi.org/10.1016/j.jcin.2012.03.005

[22] Arnold M, Achenbach S, Pfeiffer I, et al. A method to determine suitable fluoroscopic projections for transcatheter aortic valve implantation by computedtomography. J Cardiovasc Comput Tomogr. 2012; 6(6): 422-8. http://dx.doi.org/10.1016/j.jcct.2012.10.008

[23] Kurra V, Kapadia SR, Tuzcu EM, et al. Pre-procedural imaging of aortic root orientation and dimensions: comparison between X-ray angiographic planar imaging and 3-dimensional multidetector row computed tomography. JACC Cardiovasc Interv. 2010; 3(1): 105-13. http://dx.doi.org/10.1016/j.jcin.2009.10.014 